\title{
Collision Cross Sections for Protein Ions
}

\author{
T Covey and D. J. Douglas \\ Sciex, Thornhill, Ontario, Canada
}

\begin{abstract}
A method for the determination of cross sections for gas-phase protein ions, based on the energy loss of ions as they pass through a collision gas, is described. A simple model relates the energy loss to the number of collisions and hence the cross section. Results from a Monte Carlo model that support the validity of this approach are described. Experimental cross sections are reported for motilin, ubiquitin, cytochrome $c$, myoglobin, and bovine serum albumin. Cross sections range from approximately $800 \AA^{2}$ for motilin to approximately $14,000 \AA^{2}$ for bovine serum albumin and generally increase with the number of charges on the ion. Cytochrome $c$ ions from aqueous solution show somewhat smaller cross sections than ions formed from solutions of higher organic content, suggesting that the gas-phase ions may retain some memory of their solution conformation. (f Am Soc Mass Spectrom 1993, 4, 616-623)
\end{abstract}

$\mathrm{T}$ The development of electrospray and related ionization techniques has allowed, for the first time, the formation of many gas-phase protein ions [1]. Physical properties of these ions, such as size or conformation, are largely unknown. Collision cross sections can give one measure of the ion size (which may be related to conformation), but little is known about collision cross sections for these ions. Smith and Barinaga [2] reported dissociation cross sections of approximately $1000 \AA^{2}\left(10^{13} \mathrm{~cm}^{2}\right)$ for cytochrome $c$ ions. A study of collision "focusing" in radiofrequency (RF) quadrupoles led to the speculation that collision cross sections must be approximately $1000 \AA^{2}$ or more for such ions [3].

This report describes a novel method for the determination of collision cross sections for gas-phase protein ions. The loss of axial energy of an ion as it passes through a collision cell, containing an inert gas, is measured. A simple model gives the average energy loss in a single collision so that from the energy loss, the total number of collisions and hence cross section can be calculated. The use of the energy loss of ions to determine physical properties of ions (or the target) is not new (see, e.g., Bohr [4]) but to our knowledge, this is the first application to gas-phase ions of biomolecules. It is shown that for the ions studied, cross sections are approximately $10^{3}-10^{4} \AA^{2}$ and that the method may find use for studies of conformations of these ions. Described here is the experimental procedure, a simple model for the energy loss process, a Monte Carlo simulation of ion energy distributions, and collision cross sections for ions formed from

Address reprint requests to T. Covey, Sciex, 55 Glen Cameron Road, Thornhill, Ontario, Canada, L3T 1P2. motilin, ubiquitin, cytochrome $c$, myoglobin, and bovine serum albumin.

\section{Experimental}

All experiments were performed on a PE-Sciex API III triple quadrupole mass spectrometry system, shown schematically (with notation) in Figure 1. Ions formed by pneumatically assisted electrospray (ion spray) enter the vacuum chamber through a small orifice and pass through an RF quadrupole $(Q 0)$ to the first analyzing quadrupole (Q1), also operated in RF-only mode for this report. The potentials applied to the system are shown in Table 1. Ions enter the first RF quadrupole (Q0) at the potential of the orifice but have a sufficient number of collisions with the gas expanding from the orifice that their energies are moderated to a few volts or less in Q0. Therefore at Q2, the ions appear to be formed at a potential close to the $\mathrm{Q} 0$ rod offset. Because the potential difference between the $\mathrm{Q} 0$ and the collision cell $\left(Q_{2}\right)$ rod offset voltages was $10 \mathrm{~V}$, ions entered the collision cell with an energy of approximately $10 i \mathrm{eV}$, where $i$ is the number of charges on the ion (center-of-mass energies were typically $0.1-0.8 \mathrm{eV}$ ). With no collision gas added, stopping potentials were $10 \pm 0.5 \mathrm{~V}$, in accord with this interpretation. Under these condilions, no collision-induced dissociation was seen. Quadrupole Q3 was operated in mass-resolving mode. Energy distributions of ions leaving Q2 were determined approximately from stopping curves obtained by increasing the $Q 3$ rod offset voltage in steps of $1.0 \mathrm{~V}$ until the ion signal was attenuated by approximately three orders of magnitude. Ion energy spreads (at $10 \%$ ) were generally approximately $1 \mathrm{eV}$; exceptions were some ions produced from cytochrome $c$ (see 


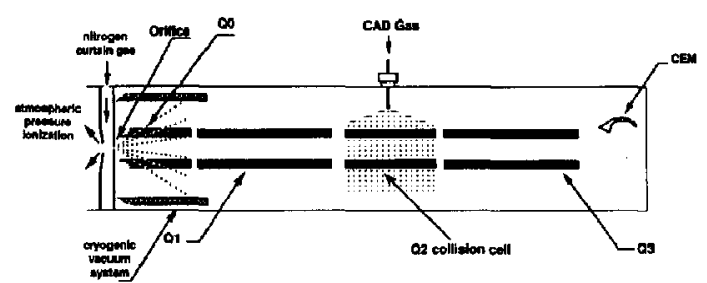

Figure 1. Triple quadrupole system. Ions enter the vacuum system through an orifice and enter an RF-only quadrupole (Q0). The first analyzing quadrupole is Q1, the collision cell an RF quadrupole $Q 2$, and the second analyzing quadrupole Q3. CAD, collision-activated dissociation; CEM channel electron multiplier.

below). Stopping curves were obtained for several values of target thicknesses. Cross sections were determined from the variation of nominal ion energy with target thickness, as described below. The target thickness in $\mathrm{Q} 2$ was calculated from the gas flow to the collision cell (at approximately $-30^{\circ} \mathrm{C}$ ) measured with an electronic flow meter (Matheson 8112-0421, East Rutherford, NJ).

Use of the $\mathrm{Q} 3$ rod offset to stop ions gives only an approximate energy distribution. More accurate distributions and smaller differences in distributions (corresponding to smaller differences in collision cross sections) could likely be observed with a proper triple-grid energy analyzer or other analyzing device. Whereas a better energy analyzer would be preferred, in this first study use of the Q3 rod offset was found to be adequate to demonstrate the energy loss method. Errors in absolute cross sections derived this way can arise from (1) the use of finite step sizes to approximate the energy distribution; (2) uncertainties in the target thickness; (3) uncertainties in the ion trajectory path length [in analyzing the data the cell length $(15 \mathrm{~cm})$ was used]; (4) approximations to the average energy calculated from the stopping data; and (5) interpretation of the data with a simple collision model (see below). All of these systematic errors can contribute to the absolute cross-section measurements. Relative cross-section measurements, however (in some ways of greater interest), should be much less subject to these sources of error.

Reagents were the following: (1) motilin (porcine, PGA 250A) (Bachem, Philadelphia, PA) $1.1 \times 10^{-5} \mathrm{M}$ in $1: 1$ acetonitrile:water, $0.1 \%$ acetic acid; (2) ubiquitin (bovine red cell, U-6253) (Sigma, St. Louis, MO) $6 \times$ $10^{-5} \mathrm{M}$ in $1: 1$ acetonitrile:water, $0.1 \%$ acetic acid; (3) cytochrome $c$ (bovine heart type V-A, C-2037) (Sigma), $4 \times 10^{-5} \mathrm{M}$ in 1:1 acetonitrile:water, $0.05 \%$ formic acid,

Table 1. Voltages used in this report

$\begin{array}{lc}\text { Orifice } & 35 \mathrm{~V} \\ \text { Q0 rod offset } & 30 \mathrm{~V} \\ \text { Q1 rod offset } & 29 \mathrm{~V} \\ \text { Q2 rod offset } & 20 \mathrm{~V} \\ \text { Q3 rod offset } & \text { Varied }\end{array}$

and $4 \times 10^{-5} \mathrm{M}$ in $1: 9$ acetonitrile:water $0.01 \%$ acetic acid; (4) myoglobin (horse skeletal muscle, M-0630) (Sigma), $2.4 \times 10^{5} \mathrm{M}$ in 1:1 acetonitrile:water, $0.1 \%$ acetic acid; and (5) bovine serum albumin ( $A-4378)$ (Sigma), $7.5 \times 10^{-5} \mathrm{M}$ in 2:8 acetonitrile:water $0.05 \%$ acetic acid. 'The collision gas was argon in all experiments.

\section{Collision Model}

The ratio of laboratory (lab) energy of an ion after collision with a neutral ( $E_{\mathrm{lab}}^{\prime}$ ) to that before collision $\left(E_{\text {lab }}\right)$ is given by [5]

$$
\begin{aligned}
\frac{E_{\text {lab }}^{\prime}}{E_{\text {lab }}}= & \frac{m_{1}^{2}+m_{2}^{2}}{M^{2}}-\frac{m_{2} E_{\text {int }}}{M E_{\text {lab }}} \\
& +\frac{2 m_{1} m_{2}}{M^{2}}\left(1-\frac{E_{\text {int }} M}{E_{\text {lab }} m_{2}}\right)^{1 / 2} \cos \theta_{\mathrm{cm}}
\end{aligned}
$$

where $m_{1}$ is the ion mass, $m_{2}$ the neutral collision partner mass, $E_{\text {int }}$ the energy transferred to internal energy of the collision partners, $\theta_{\mathrm{cm}}$ the scattering angle in center-of-mass coordinates, and $M=m_{1}+$ $m_{2}$. For an elastic collision, $E_{\mathrm{int}}=0$, and eq 1 reduces to

$$
\frac{E_{\text {lab }}^{\prime}}{E_{\text {lab }}}=\frac{m_{1}^{2}+m_{2}^{2}}{M^{2}}+\frac{2 m_{1} m_{2}}{M^{2}} \cos \theta_{\mathrm{cm}}
$$

The distribution of scattering angles $\theta_{\mathrm{cm}}$ is determined by the collision energy and the interaction potential. For hard-sphere collisions, $\theta_{\mathrm{cm}}$ is distributed between $0^{\circ}$ and $180^{\circ}$ to give a uniform distribution of postcollision energies between these limits, with an average energy transfer corresponding to $\theta_{\mathrm{cm}}=90^{\circ}$. Thus, on average,

$$
\frac{E_{\mathrm{lab}}^{\prime}}{E_{\mathrm{lab}}}=\frac{\left(m_{1}^{2}+m_{2}^{2}\right)}{M^{2}}
$$

In the limit $\theta_{\mathrm{cm}}=0^{\circ}$, no energy is transferred (a grazing, near-miss collision). In the limit $\theta_{\mathrm{cm}}=180^{\circ}$, the average energy lost is twice that given by eq 3 . The experiments clearly show a loss of ion energy through collisions, so it is unlikely that on average, $\theta_{\mathrm{cm}}=0^{\circ}$. To interpret the experimental data, eq 3 (corresponding to $\theta_{\mathrm{cm}}=90^{\circ}$ ) was taken as a measure of the average energy change per collision. For a hard-sphere potential, this is valid. At the other extreme, a strongly attractive potential leading to complex formation, ions will be isotropically scattered between $0^{\circ}$ and $180^{\circ}$, again with an average scattering angle of $90^{\circ}$. Thus, use of eq 3 is valid for both highly repulsive and highly attractive potentials. It is also likely to be realistic for intermediate cases. Use of a ratio different from that of eq 3 to interpret the experimental data will give different absolute cross sections but the same relative cross sections. 
If collisions are inelastic, the energy transferred from laboratory translational to internal energy $\left(E_{\text {int }}\right)$ can vary from 0 to a maximum given by the center-of-mass energy:

$$
E_{\mathrm{cm}}=\left(m_{2} / M\right) E_{1 \mathrm{ab}}
$$

Substituting this maximum possible value for $E_{\text {int }}$ into eq 1 gives (for all scattering angles),

$$
\frac{E_{\mathrm{lab}}^{\prime}}{E_{\mathrm{lab}}}=\frac{m_{1}^{2}+m_{2}^{2}}{M^{2}}-\frac{m_{2}^{2}}{M^{2}}
$$

Equation 5 differs from eq 3 by a correction term $m_{2}^{2} / M^{2}$. For the ions studied, this correction is small. For example, for motilin $\left(m_{1}=2700\right)$, eq 3 gives a ratio of 0.9712 , whereas eq 5 gives 0.9710 , corresponding to a less than $1 \%$ difference in energy change. For heavier ions, the correction is smaller. Therefore, the effects of inelastic collisions were not included in the data analysis.

Given the average ratio of ion energies before and after a single collision, the energy loss in passing through a cell with many collisions follows simply. Define $\alpha$ as the average ratio of laboratory energy after one collision $\left(E_{1}\right)$ to that before any collisions $\left(E_{0}\right)$, given by eq 3 , that is,

$$
\alpha=E_{1} / E_{0}=\left(m_{1}^{2}+m_{2}^{2}\right) / M^{2}
$$

After two collisions, the average laboratory energy $\left(E_{2}\right)$ is given by

$$
E_{2}=\alpha E_{1}=\alpha^{2} E_{0}
$$

and after $N$ collisions,

$$
E_{N}=\alpha^{N} E_{0}
$$

Following conventional gas kinetic theory [6], the number of collisions $N$ is given by the ratio of the cell length $!$ to the mean free path,

$$
N=l / \lambda
$$

with the mean free path given by

$$
\lambda=(n \sigma)^{-1}
$$

where $n$ is the number density of gas atoms or molecules in the collision cell, and $\sigma$ is the collision cross section. Thus, the energy of an ion leaving the collision cell is given by

$$
E / E_{0}=\alpha^{\sigma n d}
$$

Letting $\alpha^{\prime}=\alpha^{-1}$, writing $\alpha^{\prime}=\exp \left(\ln \alpha^{\prime}\right)$, and defining the target thickness $S$ by $S=n l$ gives

$$
E / E_{0}=\exp ^{-\sigma S \ln \alpha^{\prime}}
$$

In this model, ions show an exponential energy loss with increasing target thickness. Fitting the experimental energy loss to eq 12 allows determination of the collision cross section. At each target thickness, the stopping potential required to attenuate the ion intensity to one-tenth was taken as a measure of the ion energy. In fitting the data to eq 12 , ratios of stopping potentials were equated to ratios $E / E_{0}$.

This model does not include any energy dependence of the collision cross section but was found to be adequate to interpret the experimental data. Each set of stopping curves covers a range of collision energies (depending on target thickness). A strongly energy-dependent cross section would be likely to give deviations from a linear fit to eq 12 . This was not seen. More refined experiments, however, might yield some information on the variation of cross section with collision energy.

\section{Monte Carlo Model}

A Monte Carlo model (previously described in ref 3) was used to further investigate the energy loss process and to assess the validity of the simple model and the experimental approach. Figure $2 \mathrm{a}-\mathrm{d}$ shows calculated energy distributions for an ion of $400 \mathrm{Da}$ passing through a 15-cm collision cell filled with argon at 0.5 , $1.0,1.5$, and 2.0 mtorr, respectively (a pressure of 1.0 mtorr at $20{ }^{\circ} \mathrm{C}$ gives a target thickness of $4.9 \times 10^{14}$ $\mathrm{cm}^{-2}$ ). The collision cross section was $50.3 \AA^{2}$ (maximum impact parameter $b_{\max }=4.0 \AA$ [3]). Figure $3 \mathrm{a}-\mathrm{d}$ shows calculated energy distributions for an ion of $16,000 \mathrm{Da}$ with a collision cross section of $2827 \AA^{2}\left(b_{\max }\right.$ $=30 \AA$ ) passing through a $15-\mathrm{cm}$ cell at the same pressures. Despite the much greater ion mass in this second case, there is still substantial energy loss because the number of collisions is much larger. At 1.00 mtorr, the 400-Da ion makes on average 2.6 collisions, whereas the 16,000 -Da ion makes on average 140 collisions.

It is also apparent from Figures 2 and 3 that the $16,000-\mathrm{Da}$ ion retains a narrow energy distribution at all cell pressures, whereas the lighter ion acquires a considerable energy spread. The spread derives from the variation in the number of collisions; if in passing through the cell there are $N$ collisions, then the variation is approximately $\sqrt{N}$. The $400-\mathrm{Da}$ ion at $1.0 \mathrm{mtorr}$ then has $2.46 \pm 1.63$ collisions, a spread in collision number of approximately $\pm 60 \%$. In contrast, the $16,000-\mathrm{Da}$ ion has $140 \pm 11.8$ collisions, a spread in collision number of only approximately $\pm 8 \%$. This narrow energy spread is a feature unique to massive ions with very large collision cross sections. Initially it was thought that to derive cross sections from the experimental data, it might be necessary to fit broad distributions with the Monte Carlo model. The narrow distributions apparent in Figure 2 (and evident from the experimental stopping curves), however, mean that the average ion energy is easily derived to a good 


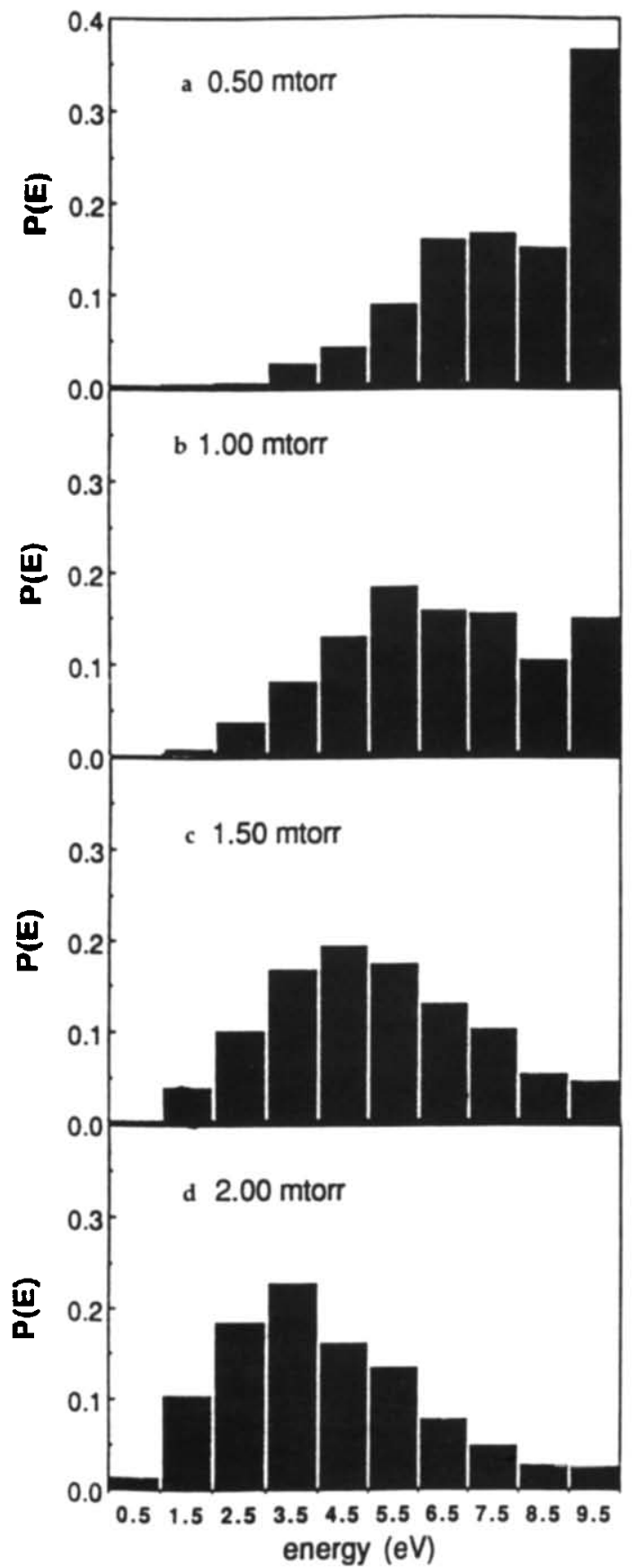

Figure 2. Energy distributions of an ion of $400 \mathrm{Da}$ with a collision cross section of $50.3 \AA^{2}$ from the Monte Carlo model. The initial ion energy was $10 \mathrm{cV}$. The energy scale is divided into 10 "bins" of $1 \mathrm{eV}$ width. P(E), potential energy.

approximation from the stopping curves without detailed fitting.

The Monte Carlo energy distributions were used to calculate average energies at each collision cell pres-

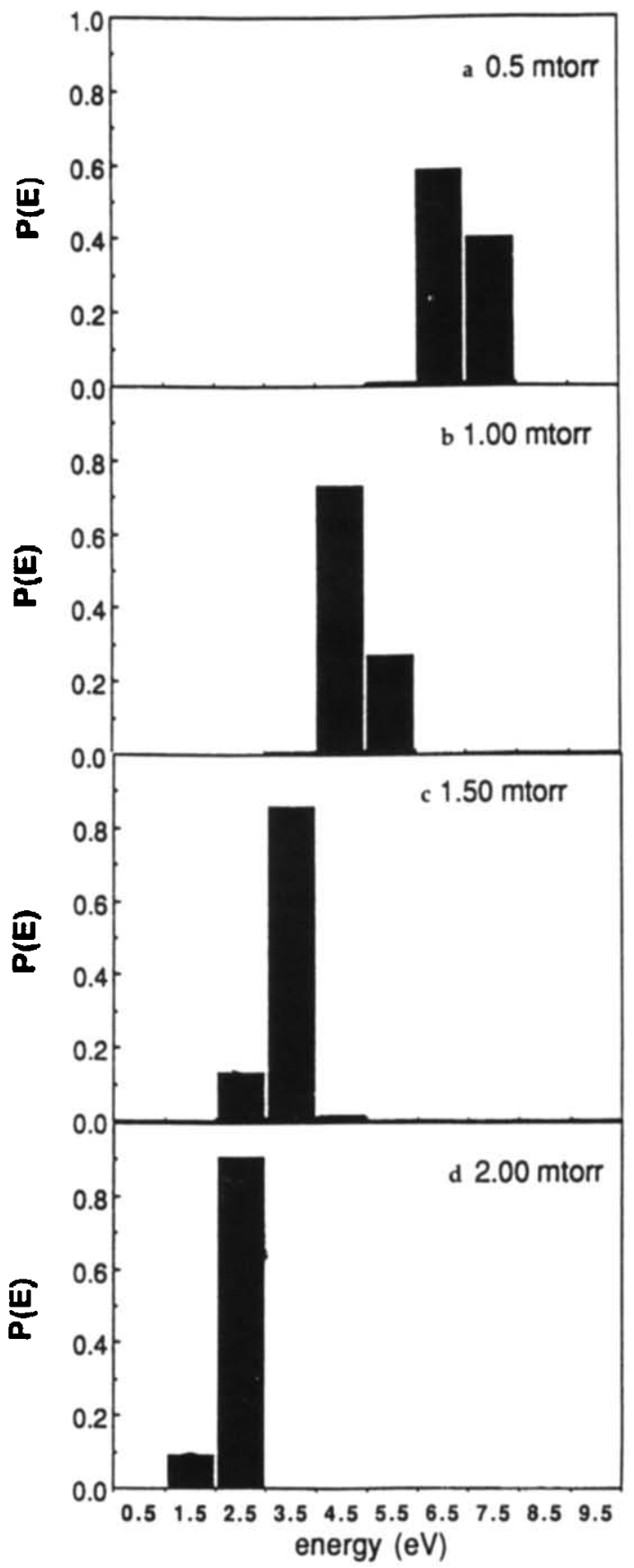

Figure 3. Energy distributions for an ion of $16,000 \mathrm{Da}$ with a collision cross section of $2827 \AA^{2}$ from the Monte Carlo madel. The energy scale is divided into 10 "bins" of $1 \mathrm{eV}$ width. $\mathrm{P}(\mathrm{E})$, potential energy.

sure. These average energies were then fit to eq 12 and the collision cross sections calculated. Ideally, the calculated cross section will agree with that used in the original Monte Carlo simulation. "Error" can arise, however, because the energy "bins" are of finite width 
and, to a lesser extent, because there is a statistical uncertainty in the number of ions in each "bin" (approximately the square root of the number of ions). From the fit, the calculated cross section for the 400-Da ion is $44 \AA^{2}$ (an "error" of $6.3 \AA^{2}$, or $12 \%$ ) and for the $16,000-\mathrm{Da}$ ion, $2676 \AA^{2}$ (an "error" of $151 \AA^{2}$, or $5 \%$ ). These "errors" can be taken as an estimate of the errors that might be incurred by using finite steps in the stopping curves.

Experimental stopping curves were generally quite sharp, showing a decrease in ion intensity of one to two orders of magnitude, with a stopping potential change of $1 \mathrm{~V}$ (e.g., Figure 4 below). In interpreting the experimental data, the average ion energy was approximated as the energy at which the ion intensity was reduced to one-tenth of the value with no stopping potential. This value was interpolated from the stopping curves and is referred to as $E_{1 / 10}$. To investigate the validity of this procedure, synthetic stopping curves were produced from the Monte Carlo energy distributions of Figure 3. The values of $E_{1 / 10}$ taken from these synthetic stopping curves were fit to eq 12 . The cross section calculated from this procedure is $2835 \AA^{2}$, in fortuitously good agreement with the $2827 \AA^{2}$ used to generate the distribution, and only $6 \%$ different from that derived from an exact evaluation of the average energies. It is concluded that the procedure of taking $E_{1 / 10}$ as a measure of the ion energy does not introduce excessive errors in the derived cross sections. Protein ions are a particularly favorable case for this procedure because the ion energy distributions remain narrow throughout the energy loss process.

\section{Results}

Results are discussed for each compound studied. Table 2 reports cross sections for all ions studied listed in order of increasing molecular weight.

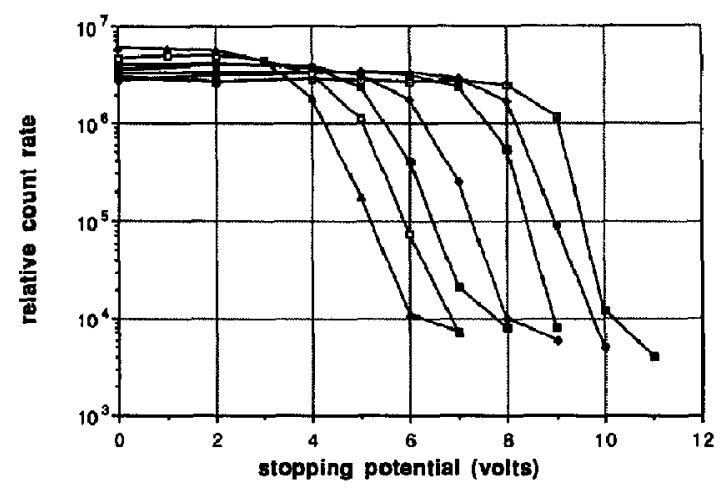

Figure 4. Stopping curves for myoglobin $\left(m / z 1542^{+},+11\right)$ at target-thickness values of $0(\square), 9.64 \times 10^{13}(\diamond), 1.47 \times 10^{14}$ (D), $2.61 \times 10^{14}(0), 3.35 \times 10^{14}(\mathbf{0}), 4.25 \times 10^{14}(\square)$, and 5.33 $\times 10^{14} \mathrm{~cm}^{-2}(\mathrm{~A})$.
Table 2. Collision Cross Sections ${ }^{\mathbf{a}}$

\begin{tabular}{|c|c|c|}
\hline$m / z$ & charge & $\begin{array}{c}\text { Cross section } \\
\qquad\left(A^{2}\right)\end{array}$ \\
\hline \multicolumn{3}{|c|}{ Motilin (MW 2699) } \\
\hline 901 & 3 & 850 \\
\hline 676 & 4 & 890 \\
\hline 541 & 5 & 950 \\
\hline \multicolumn{3}{|c|}{ Ubiquitin (MW 8564) } \\
\hline 1224 & 7 & 1460 \\
\hline 952 & 9 & 1900 \\
\hline 779 & 11 & 2220 \\
\hline \multicolumn{3}{|c|}{ Cytochrome $c$ (MW 12,231 ) in 9:1 water:acetonitrile } \\
\hline 1748 & 7 & 1450 \\
\hline 1530 & 8 & 1800 \\
\hline 1360 & 9 & 1800 \\
\hline 1224 & 10 & 2020 \\
\hline \multicolumn{3}{|c|}{ Cytochrome $c$ (MW 12,231) in 1:1 water:acetonitrile } \\
\hline 1360 & 9 & 2370 \\
\hline 1224 & 10 & 2430 \\
\hline 1113 & 11 & 3230 \\
\hline 1020 & 12 & 3230 \\
\hline 875 & 14 & 3830 \\
\hline 765 & 16 & 3450 \\
\hline 680 & 18 & 4120 \\
\hline 612 & 20 & 4310 \\
\hline \multicolumn{3}{|c|}{ Myoglobin (MW 16.950) } \\
\hline 2120 & 8 & 2520 \\
\hline 1884 & 9 & 2570 \\
\hline 1542 & 11 & 3020 \\
\hline 1305 & 13 & 3550 \\
\hline 1131 & 15 & 4040 \\
\hline 998 & 17 & 4290 \\
\hline 893 & 19 & 4820 \\
\hline 808 & 21 & 5040 \\
\hline \multicolumn{3}{|c|}{ Bovine serum albumin (MW 66,431) } \\
\hline 2215 & 30 & 11,400 \\
\hline 1846 & 36 & 13,300 \\
\hline 1749 & 38 & 13,700 \\
\hline 1621 & 41 & 14.000 \\
\hline
\end{tabular}

${ }^{a}$ MW, molecular weight.

\section{Myoglobin}

The mass spectrum of myoglobin is similar to that obtained in ref 7 . This was the first compound studied and is considered typical. Stopping curves were obtained for protonated molecules with charges $8,9,11$, $13,15,17,19$, and $21(\mathrm{~m} / \mathrm{z} 2120,1884,1542,1305,1131$, 998,893 , and 808, respectively). Representative stopping curves, those for $m / z 1542$ ( +11 ions) are shown in Figure 4. All stopping curves show a sharp decrease of one to two orders of magnitude, with a potential change of $1 \mathrm{~V}$ on Q3. This is consistent with the narrow energy distributions throughout the energy loss process, seen in the Monte Carlo model. The $E_{1 / 0}$ energies for myoglobin +11 at each target thickness and an exponential fit to the data are showr in Figure 5. Shown also are the data and fits for +21 ions $(\mathrm{m} / \mathrm{z}$ 808) and +15 ions ( $m / z$ 1131). It is apparent that ions of different charge state show different energy losses despite being of nearly equal mass. These differences in energy loss derive from different collision cross sections. The quality of the fits in Figure 5 are typical 


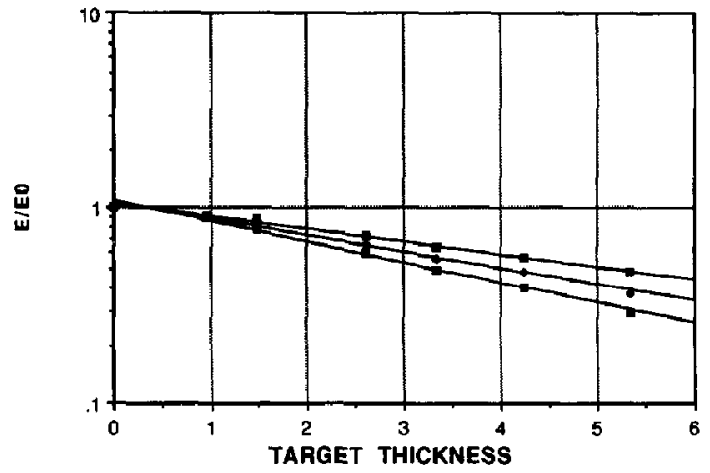

Figure 5. Ratio of ion entergy ( $E$ ) lo ion energy wilh nu collision gas added ( $E_{0}$ ) versus target thickress (in units of $10^{14} \mathrm{~cm}^{-2}$ ) for myoglobin: $m / z 808(+21)(\square), m / z 1131(+15)(\uparrow)$, and $m / z$ $1542(+11)(\square)$. Solid curves, exponential fits to the data.

of those seen for most ions. Cross sections for all of the myoglobin ions studied are shown in Table 2 . There is an approximate linear increase in collision cross section with increasing charge (Figure 6).

\section{Cytochrome $c$}

Two cytochrome $c$ solutions were run: $4 \times 10^{-5} \mathrm{M}$ cytochrome $c$ in 9:1 wateracelonilrile, and $4 \times 10^{-5} \mathrm{M}$ cytochrome $c$ in 1:1 water:acetonitrile (referred to as high-aqueous and high-organic solutions, respectively). The high-aqueuus solution produced cytochurome $c$ ioms in low-charge states, +7 to +10 ; the high-organic solution produced ions in higher charge states, +9 to +20 . (Mass spectra were similar to those in ref 8.) These differences in charge distributions have been interpreted as arising from different protein conformations in solution, the protein being denatured in the high-organic content solution [8, 9]. Ions formed from the two solutions showed qualitatively different stopping curves.

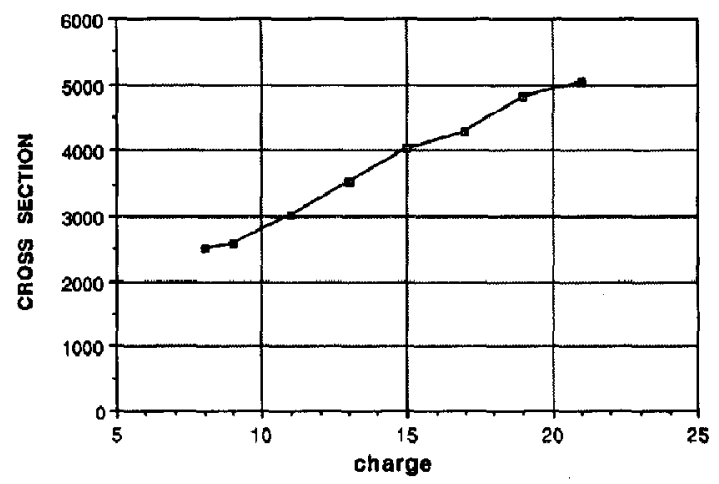

Figure 6. Cross section $\left(\AA^{2}\right)$ versus charge for protonated myoglobin ions.
Cytochrome $c$ in 9:1 Water:Acetonitrile. Stopping curves for ions from cytochrome $c$ in 9:1 water:acetonitrile were similar to those obtained from myoglobin (Figure 4), showing a sharp decrease in signal intensity with approximately $1 \mathrm{~V}$ of stopping potentials. Exponential fits to $E_{1 / 10}$ data were also of similar quality. Cross sections for the +7 to +10 ions ( $m / z 1748,1530,1360$, and 1224, respectively) are reported in Table 2 .

Cytochrome $c$ in 1:1 Water:Acetonitrile. Stopping curves for ions from cytochrome $c$ in high-organic solution were appreciably broader than those for all other ions studied. An example is shown in Figure $7(\mathrm{~m} / z 680$, +18 ). Even with no added collision gas, the ion energy distribution is apparently broader. Ions at $m / z 1360$ and $1224(+9$ and +10 , respectively) showed broader energy distributions than those of the same ions formed from the high-aqueous solution. This broadening makes interpretation of the nominal ion energy more difficult, but the $E_{1 / 10}$ energies are still used as a measure of ion energy. Exponential fits to the data are generally of poorer quality (the poorest of all ions studied). As an example, the fits for ions of $m / z 680$, 1020 , and 1360 are shown in Figure 8. Cross sections for the ions studied are shown in Table 2. Figure 9 shows the cross sections for all cytochrome $c$ ions studied (both solutions) plotted against their charge. As with myoglobin, there is a general increase in collision cross section with charge. Ions formed from the high-aqueous solution show somewhat smaller cross sections than the same ions formed from the high-organic solution and also somewhat smaller cross sections than those expected from extrapolation from the higher charge states observed with the high organic content solution.

\section{Motilin}

This compound was included as an example of a smaller peptide. Sharp stopping curves and good exponential fit were obtained. Cross sections for ions

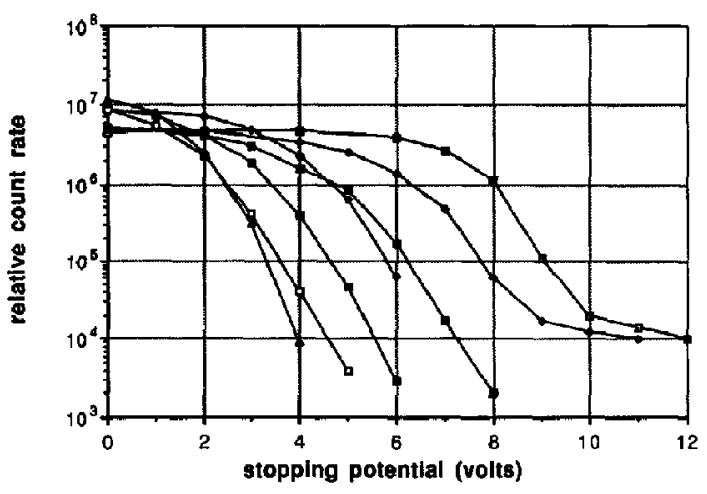

Figure 7. Stopping curves for cytochrome $c\left(m / z 680^{+},+18\right.$ at target-thickness values of $0\left(0^{\circ}\right), 8.51 \times 10^{13}(\bullet), 1.76 \times 10^{14}$ (a) $2.32 \times 10^{14}(\diamond), 2.95 \times 10^{14}, 4.14 \times 10^{14}(\square)$, and $4.93 \times$ $10^{14} \mathrm{~cm}^{-2}(\Lambda)$ 


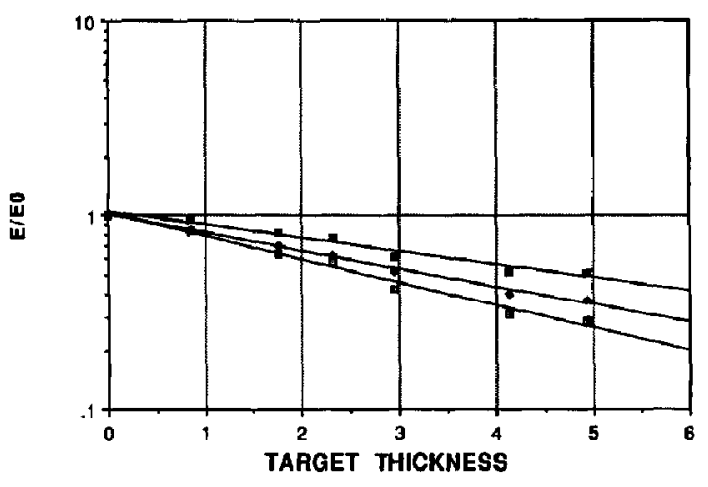

Figure 8. Ratio of ion energy $(E)$ to ion cnergy with no collision gas added $\left(E_{0}\right)$ versus target thickness (in units of $10^{14} \mathrm{~cm}^{-2}$ ) for cytochrome c: $m / z$ $680^{+}(+18)(\square), m / z 1020(+12)(\$)$, and $m / z 1360(+9)$ (D). Solid curves, exponential fits to the data.

with charges $+3,+4$, and $+5(\mathrm{~m} / \mathrm{z} 901,676$, and 541 , respectively) are reported in Table 2.

\section{Ubiquitin}

Again, sharp stopping curves and good exponential fits were found. Cross sections for ions with charges +7 , +9 , and $+11(m / z 1224,952$, and 779 , respectively) are shown in Table 2 .

\section{Bovine Serum Albumin}

Bovine serum albumin was the highest molecular weight protein studied. Sharp stopping curves and good exponential fits were found. Cross sections for ions with charges $+30,+36,+38$, and $+41(\mathrm{~m} / \mathrm{z}$ $2215,1846,1749$, and 1621 , respectively) are reported in Table 2.

\section{Discussion}

The most immediate result apparent from the cross sections shown in Table 2 is that they are very large compared with collision cross sections of small organic

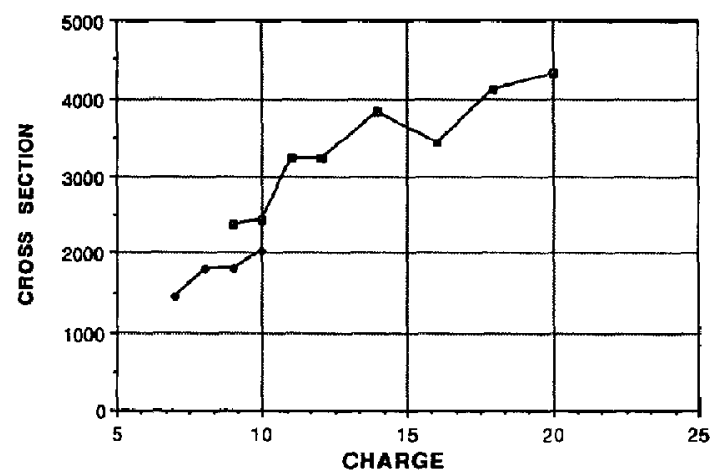

Figure 9. Cross sections $\left(\AA^{2}\right)$ versus charge for cytochrome $c$ ions from 9:1 water:acetonitrile $(\bullet)$ and 1:1 wateracetonitrile solutions (๑). ions (typically $10-100 \AA^{2}$ ). Perhaps this is not surprising for protein ions, but to our knowledge, this is the first direct measurement of these large cross sections. It is also apparent that the cross sections increase with charge state for all of the ions studied. To some extent, this might be expected from a larger ion-induced dipole attractive force between the more highly charged ions and the argon collision partner. For a multiply charged ion, this force leads to an energy-dependent Langevin collision cross section $\sigma_{1}[10]$,

$$
\sigma_{1}=\frac{(i) 2 \pi}{v_{0}}\left(\frac{e^{2} \beta}{M_{r}}\right)^{1 / 2}
$$

where $i$ is the number of charges on the ion, $e$ the electron charge, $v_{0}$ the relative velocity of the collision partners, $M_{\mathrm{r}}$ the reduced mass of the collision partners $\left[M_{\mathrm{r}}=\left(m_{1} m_{2}\right) /\left(m_{1}+m_{2}\right)\right]$, and $\beta$ the polarizability of the argon atom $\left(1.65 \times 10^{-24} \mathrm{~cm}^{3}[11]\right)$. In the experiments reported here, the incident ion energy (i.e., before collisions) was proportional to charge, so the initial relative velocity varies as $\mathrm{v}_{0} \approx i^{1 / 2}$. Thus, in the Langevin model, the observed collision cross sections should vary as $i^{1 / 2}$. In fact, a near-linear increase with charge is seen (Figures 6 and 9). Stopping potentials for ions generally ranged from approximately $10 \mathrm{~V}$ in the absence of collision gas to approximately $3 \mathrm{~V}$ at the highest target gas densities corresponding to laboratory collision energies ranging from approximately $10 i$ $\mathrm{eV}$ down to $3 i \mathrm{eV}$. Absolute cross sections calculated from eq 13 for an incident ion energy of $10 i \mathrm{eV}$ are five to eight times smaller than observed, and for ion energies of $3 i \mathrm{eV}$ are three to four times smaller than observed. The I angevin model, then, does not account for the experimental cross sections. A larger ionneutral attractive force may lead to larger collision cross sections for more highly charged ions, but this cannot be the only factor. It seems plausible that the larger cross sections for ions in higher charge state result from a more open, extended structure of the proteins, possibly from Coulombic repulsion of the charges. (A reviewer has noted that the X-ray-determined dimension of myoglobin is $44 \AA \times 44 \AA \times$ $25 \AA$ to give a cross section of approximately $1100-1940$ $\AA^{2}$, depending on orientation, and that the cross sections shown in Table 2 are therefore reasonable, allowing for an increase with charge state.)

For cytochrome $c$, the collision cross sections for ions formed from the more highly aqueous solution are significantly smaller than those formed from the more organic solution. The charge distributions are consistent with a more open conformation in the organic solution and a more closed conformation in the aqueous solution, as described by others $[8,9,12,13]$. The experimental cross sections suggest that the gasphase ions retain some memory of the solution conformation, giving "smaller" ions from the aqueous solution and "larger" ions from the organic solution. 
The energy loss method described here may be of use in further studies of conformations of gas-phase proteins. Different protein conformations need not necessarily give different collision cross sections. For example, a rod-shaped protein ion can give either a larger or smaller cross section than a spherical protein ion, depending on orientation. The collision cross sections are an average of over all orientations. Nevertheless, in favorable cases, and with more refined energy measurements, conformation information should be possible.

\section{References}

1. Fenn, J. B.; Mann, M.; Meng, C. K.; Wong, S.F.; Whitehouse, C. M. Science, 1989, 246, 64 .

2. Smith, R. D.: Barinaga, C. J. Rapid Commun. Mass Spectrom. 1990, 4,54 .

3. Douglas, D. J.; French, J. B. J. Am. Soc. Mass Spectrom. 1991, 3,398 .
4. Bohr, N. Philos, Mag. 1913, 25, 10.

5. Douglas, D. J. J. Phys. Chem. 1982, 56, 185-191.

6. Kauzmann, W. Kinetic Theory of Gases; W. A. Benjamin: New York, 1966.

7. Covey, T. R.; Bonner, R. F.; Shushan, B. I. Rapid Commun. Mass Spcctrom. 1988, 2, 249.

8. Chowdury, S. K.; Katta, V; Chait, B. J. I. Am Chem. Soc. $1990,112,9012$.

9. Loo, J.A.; Loo, R. R. O; Usdeth, H. R.; Edmonds, C. G.; Smith, R. D. Rapid Commun. Mass Spectrom. 1991, 5, 101.

10. McDaniel E. W. Collision Phenomenta in Ionized Gases; John Wiley: New York, 1964; p. 72.

11. Gombas, P. In Handbuch der Physik, vol. 36; Flugge, S. Ed.; Springer-Verlag: Berlin 1956; p. 192.

12. Feng, R.; Konishi Y. Proceedings of the 40th ASMS Conference on Mass Spectrometry and Allied Topics; Washington, DC, May 31-June 5, 1992.

13. Suckau, D; Shi, Y.; Quinn, J. P.; Senko, M. W.; Zhang, M.; McLafferty, F. W. Proceedings of the 40th ASMS Conference on Mass Spectrometry and Allied Topics; Washington, DC, May 31-June 5, 1992. 